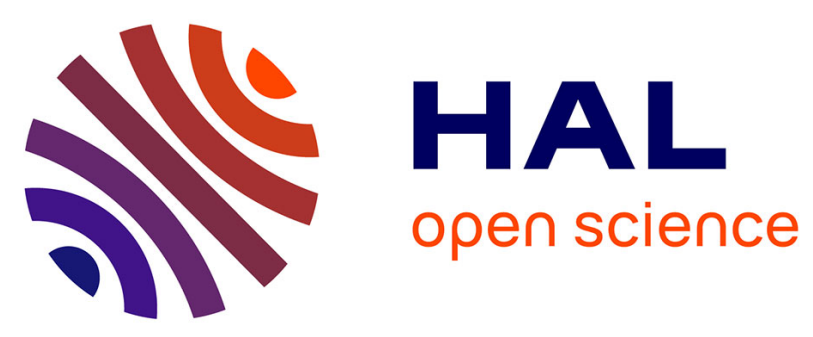

\title{
JOINT DIRECTION AND VOLUME TOMOGRAPHICAL AB-INITIO RECONSTRUCTION FOR ELECTRON MICROSCOPY
}

Bassem Ben Cheikh, Etienne Baudrier, Gabriel Frey

\section{- To cite this version:}

Bassem Ben Cheikh, Etienne Baudrier, Gabriel Frey. JOINT DIRECTION AND VOLUME TOMOGRAPHICAL AB-INITIO RECONSTRUCTION FOR ELECTRON MICROSCOPY. 12th International Symposium on BIOMEDICAL IMAGING: From Nano to Macro, Apr 2015, New-York, United States. pp.1040-1043, 10.1109/ISBI.2015.7164049 . hal-01314398

\author{
HAL Id: hal-01314398 \\ https://hal.science/hal-01314398
}

Submitted on 11 May 2016

HAL is a multi-disciplinary open access archive for the deposit and dissemination of scientific research documents, whether they are published or not. The documents may come from teaching and research institutions in France or abroad, or from public or private research centers.
L'archive ouverte pluridisciplinaire HAL, est destinée au dépôt et à la diffusion de documents scientifiques de niveau recherche, publiés ou non, émanant des établissements d'enseignement et de recherche français ou étrangers, des laboratoires publics ou privés. 


\title{
JOINT DIRECTION AND VOLUME TOMOGRAPHICAL AB-INITIO RECONSTRUCTION FOR ELECTRON MICROSCOPY
}

\author{
Bassem Ben Cheikh $\quad$ Étienne Baudrier $\quad$ Gabriel Frey ${ }^{\star}$ \\ ${ }^{\star}$ University of Strasbourg, CNRS ICube 300 Bd S. Brant - BP 10413 - F-67412 ILLKIRCH, FRANCE \\ † Sorbonne Universités, UPMC Univ Paris 06, CNRS, INSERM, LIB, F-75013, Paris, France \\ Email: bassem.bencheikh@lib.upmc.frｂaudrier@unistra.frｇ.frey@unistra.fr
}

\begin{abstract}
This paper deals with the problem of three-dimensional volume reconstruction from two-dimensional projections with unknown orientations. This situation occurs in cryo-electron microscopy. A method determining the orientations and reconstructing the volume jointly from scratch (ab initio) is presented in this work. It is based on a heuristic cost minimization including a comparison of the input projections with the projections obtained from the image being reconstructed and the orientations information and a data fitting based on the common-lines. The method is tested on synthetic data and compared to SIMPLE software on real data.
\end{abstract}

Index Terms - Tomography, Ab-initio Reconstruction, Unknown Directions, 2D, Gray-level Image.

\section{INTRODUCTION AND STATE OF THE ART}

The problem of tomographic reconstruction from projections with unknown directions is encountered in various domains as medical imaging (e.g. when the patient is moving during a X-ray scanner acquisition) and cryo electron microscopy (cryo-EM). In cryo-EM, a thin vitreous ice layer containing multiple specimens is magnified with an electron microscope. Each cryo-EM image is a $2 \mathrm{D}$ projection of the $3 \mathrm{D}$ scattering density. Because the orientations of the 3D specimens relative to the microscope are not known, it follows that each image is related to an unknown-orientation $2 \mathrm{D}$ projection of the $3 \mathrm{D}$ specimen. In fact, unlike what is essentially done in medical imaging, cryo-EM don't allow to rotate the 3D specimen and to take a series of images with known relative orientations because the specimen is rapidly damaged by the electron beam. Therefore, taking multiple images of one specimen in different orientations is replaced by taking one image of many identical specimens where each specimen is in a different random unknown orientation.

The image to reconstruct is a 3D image. Generally, in the first step, the projection directions are estimated. In 3D, it relies on algorithms derived from common line correlation [1]. If the molecule is known to have some preferred orientation, then it is possible to find an ab initio 3D structure using the random conical tilt method [2]. It exists solutions to the ab initio estimation problem of the 3D structure that do not involve tilting [3], [4]. To cope with the high dimensional space of the data, the direction-assignment algorithms use dimension reduction [5] or optimization [6, 7]. To reduce variability, class averages are typically computed from particle images that have already been rotationally and translationally aligned [8]. The choice of reference images for the alignment is, however, arbitrary and can represent a source of bias in the classification process. Ideally one would want to do the $3 \mathrm{D}$ reconstruction directly from projections in the form of raw images. This therefore sets the goal for an ab initio reconstruction algorithm that requires as little averaging as possible.

Once the direction estimation is done, the second step is the reconstruction with known directions. The main families of reconstruction methods are [2]: the algebraic methods, iterative filtered back projection, and those using the Fourier transform. In the ab-initio case, the sequence of these two steps gives a first reconstructed image whose quality is not always sufficient, and then requires a refinement step. The reconstructed image is used to refine the projection directions and these directions can then be used to improve the data reconstruction. This iteration reflects the fact that if the estimated directions influence the image reconstruction, the image estimation also affects the estimation of directions. But this does not allow the estimated image to play its full role in the estimation of directions, since it was built later. We propose a method that offers this possibility. It is based on a joint estimation of projection directions and image.

The novelty of our method lies in the fact that the image and directions as a single object to reconstruct and that they are jointly estimated. It generalizes our article focused on 2D gray-level image reconstruction [9].

The rest of the paper is structured as follows: in Sec. 2, a model of the acquisition is shown and the problem is specified, the resolution method is detailed and the associated cost function is studied then results are given in Sec. 3. A conclusion and perspectives end this paper. 


\section{PROBLEM AND METHOD}

In cryo-EM, 2D images modeled as projections of a 3D single object in different directions are provided. We assume here that the shortcomings of the acquired data (defocusing, contrast transfer function, aberrations) have been corrected during a pre-processing step. These projections come down from the X-ray transform of the object. We first introduce some necessary notations before defining the $\mathrm{X}$-ray transform.

Let $\mathbb{B}_{3}=\left\{x \in \mathbb{R}^{3} \mid\|x\| \leq 1\right\}$ be the unit ball of $\mathbb{R}^{3}$. We call $\mathcal{K}$ the set of the bounded measurable functions $f$ (for the usual measure of Lebesgues) from the unit ball $\mathbb{B}_{3}$ in $\mathbb{R}^{+}$such that $f$ is derivable and $\|\nabla f\|_{1}=\int_{\mathbb{B}_{3}}|\nabla f|<\infty$.

Remark 2.1. As $f$ is bounded, $\|f\|_{2}=\int_{\mathbb{B}_{3}}|f|^{2}<\infty$.

Definition 2.2 (X-ray transform). Let $f$ a function of $\mathcal{K}$. The $\mathrm{X}$-ray transform of $f$ is given by

$$
X_{f}(\theta, s)=\int_{\mathbb{R}} f(s+u \cdot \theta) d u
$$

where $\theta \in S^{3}$ and $s \in \theta^{\perp}$.

The function $f$ is called object and the measurable function $s \mapsto X_{f}(\theta, s)$ is called projection of $f$ in the direction $\theta$ and denoted $\pi_{2}(\theta, f)$ or $\pi(\theta, f)$ if there is no ambiguity.

$\pi(\theta, f)$ belongs to the set of bounded measurable functions with support in $\mathbb{B}_{2}$, denoted $\mathcal{B}$. The projections have the following property that will be exploited for the reconstruction.

Proposition 2.3 (Common line). Let $\pi_{2}\left(\theta_{1}, f\right)$ and $\pi_{2}\left(\theta_{2}, f\right)$ two projections of the function $f$. Let $d=\theta_{1} \wedge \theta_{2}, \gamma_{12}=$ $d^{\perp} \cap \theta_{1}^{\perp}$ and $\gamma_{21}=d^{\perp} \cap \theta_{2}^{\perp}$. Then one has the following equality:

$$
\pi_{1}\left(\gamma_{12}, \pi_{2}\left(\theta_{1}, f\right)\right)=\pi_{1}\left(\gamma_{21}, \pi_{2}\left(\theta_{2}, f\right)\right)
$$

where $\pi_{1}(\alpha, g)$ is the $1 D$ projection of the $2 D$ function $g$ in the direction $\alpha$.

Practically, the planes of projections associated to the two images intersect in a line $d$ called the common line; see Fig. 1 for an illustration.

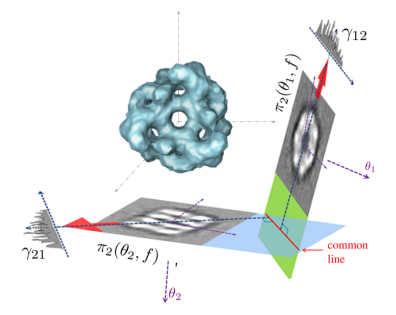

Fig. 1. Common line principle on two projections

Hence, the 1D orthogonal projections of the two images onto the common line are equal. Note that there is a version of the common line property in the Fourier's space.
Our proposal for reconstructing a 3D function $f$ (standing for a density electron map in the cryo-EM frame) from a set of its $2 \mathrm{D}$ projections $\left(P_{1}, \ldots, P_{n}\right)$ with unknown directions is to solve the following minimization problem:

$$
(\tilde{f}, \tilde{\Theta})=\underset{f, \Theta}{\operatorname{argmin}} G_{\boldsymbol{P}}(f, \Theta)
$$

where

$$
G_{\boldsymbol{P}}(f, \Theta)=\beta \boldsymbol{J}_{\boldsymbol{P}}(f, \Theta)+(1-\beta) K_{\boldsymbol{P}}(\Theta)
$$

\section{$\beta \in] 0,1]$ is a weight factor.}

The cost function $G_{\boldsymbol{P}}$ is based on three elements which are the current image, current directions and the given projections. $G_{P}$ is formed of a residual norm $\boldsymbol{J}_{\boldsymbol{P}}$ between the given projections and the estimated projections and an attach to the data $K_{P}$ depending only on the given projections and the current directions. Implementation of (2) relies on the definition of the cost function $G_{\boldsymbol{P}}$ and on an optimization method. In this paper, $\boldsymbol{J}_{\boldsymbol{P}}$ is classically formed of a residual norm that estimates an error between the given projections $\boldsymbol{P}$ and current projections $\left\{\pi\left(\theta, f^{c r t}\right), \theta \in \Theta^{\text {crt }}\right\}$ which are two finite subsets of $\mathcal{B}$ :

$$
\boldsymbol{J}_{\boldsymbol{P}}(f, \Theta)=\sum_{i=1}^{n}\left\|P_{i}-\pi_{2}\left(\theta_{i}, f\right)\right\|_{2}^{2}
$$

and $K_{\boldsymbol{P}}$ gives a direction consistency based on the common line property

$$
K_{\boldsymbol{P}}(\Theta)=\sum_{i=1}^{n} \sum_{j=i+1}^{n}\left\|L_{i, j}-L_{j, i}\right\|_{2}^{2},
$$

where

$$
L_{i, j}=\pi_{1}\left(\gamma_{i j}, P_{i}\right), \quad \forall i, j \in 1, . ., n
$$

To calculate the minimum of the cost function $G_{\boldsymbol{P}}$ we use the metaheuristic optimization algorithm, the Simulated Annealing, that gives good results to our work in the 2D case [9]. Since this method is an ab initio reconstruction method, the algorithm is initialized with an empty cube and an initial temperature $T=T_{0}$. At each iteration an elementary modification of the system is applied and evaluated by the variation of cost function. If the variation is negative, the proposed modification is accepted. Otherwise, it is accepted with a probability $e^{\frac{-\Delta G}{T}}$. The temperature $T$ decreases at each iteration toward zero following some annealing schedule. The elementary modification of $(\Theta, f)$ is based on: (1) a modification in the object $f$ by picking randomly a voxel and assigning to it a random value. (2) a modification in the set $\Theta$ by selecting randomly a projection and assigning to it a random direction. The process is stopped when the modifications are rejected $n_{0}$ times. Different types of temperature decay functions have been tested. The exponential decreasing functions give the 
better results with low decay rates $\lambda=10^{-3}$ and high initial temperatures $T_{0}=100$. The algorithm of the minimization process is shown in Algo 1.

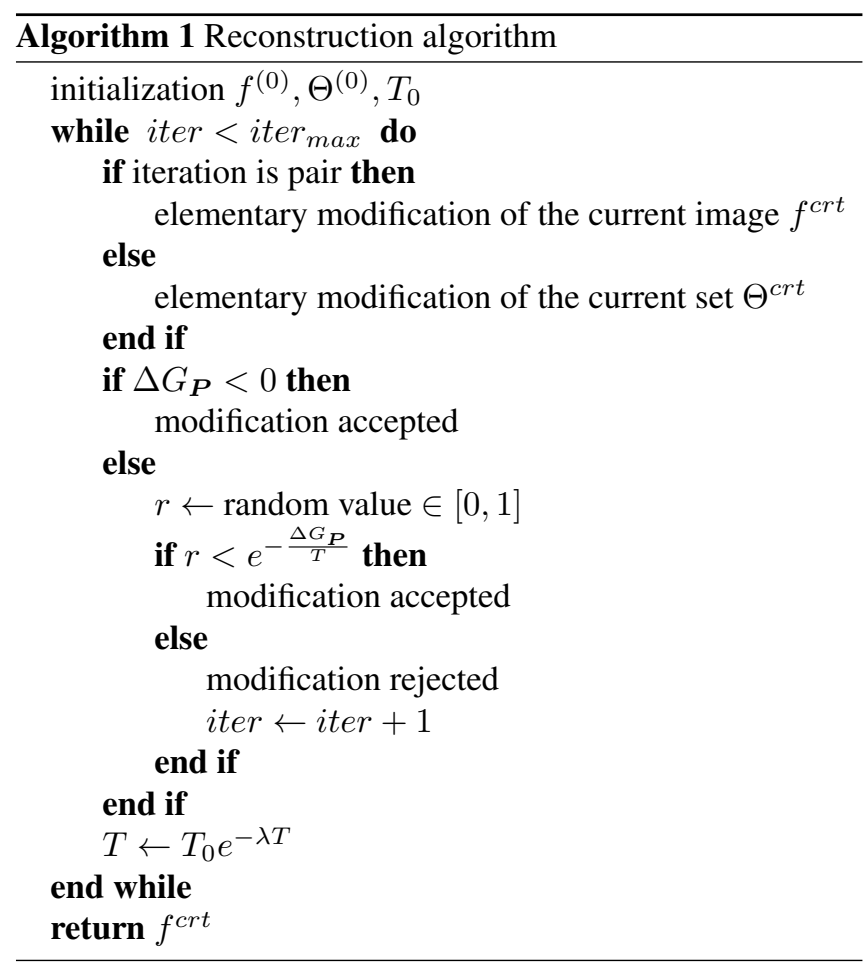

One of the most important parameters of the algorithm is the weight factor $\beta$. The best value is experimentally determined as $\beta=0.2$. Table 1 shows the different reconstruction errors depending on this parameter. Note that the two terms of $G_{\boldsymbol{P}}$ are normalized with the number of projections, the image size and $\max \left(P_{i}\right)$ to have a meaningful balance with $\beta$.

\begin{tabular}{|c|c|c|c|c|c|c|}
\hline$\beta$ & 0 & 0.2 & 0.4 & 0.6 & 0.8 & 1 \\
\hline MSE $f(\%)$ & $n . a$. & $\mathbf{0 . 9}$ & 1.9 & 1.8 & 2.4 & 6.7 \\
\hline MSE $\Theta(\%)$ & 3.1 & $\mathbf{2 . 2}$ & 2.6 & 2.6 & 3.2 & 8.4 \\
\hline
\end{tabular}

Table 1. The reconstruction quality in function of $\beta$.

The fact that the best value for $\beta$ is not 0 means that estimate the directions only from the projections does not give the best results. A consequence is that the choice of reconstruct first the directions and secondly the image (as in the classical methods) does not lead to the best reconstruction. It justifies our proposal of a joint reconstruction of the object and the directions.

\section{RESULTS}

The proposed method have been applied to 20 gray-level 3D images of different resolutions $\left(\mathrm{m}^{3}, m \in 16,32,64,128\right)$ and different gray-level numbers $\left(2^{p}, p \in 1, . ., 8\right)$, referred to as phantom images (an example is given Fig. 2).
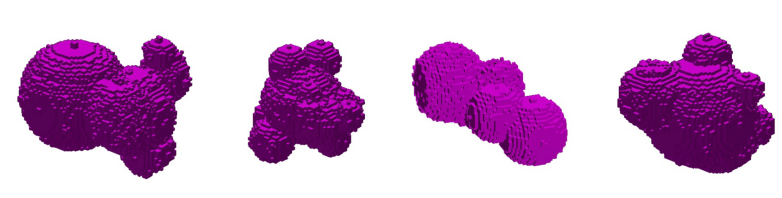

Fig. 2. Example of a phantom from the test database.

The phantoms are generated randomly by a Matlab program $^{1}$. For each resolution $m$, a set of $n_{p}=1.7 \times m$ projections uniformly distributed have been computed from the phantoms. A random direction have been assigned to each projections. The importance of the different parameters (initial temperature, temperature decay, acceptance probability, length of each reconstruction phase) has been evaluated extensively, but the study can not be shown here due to the lack of place. The parameters used for the simulation come from a compromise between the reconstruction quality and the process time. For each of the 20 phantoms, 20 reconstructions have been carried out and only the one with the minimum cost value is retained. The reconstructed volumes have been compared to the phantoms and evaluated in term of Mean Square Error (MSE) after rigid registration. The MSE is normalized with the number of gray-scale and the volume of the sphere inscribed in the image support:

$$
M S E=\frac{3}{n_{g} 4 \pi\left(\frac{N}{2}\right)^{3}} \sum_{i=1}^{N} \sum_{j=1}^{N} \sum_{k=1}^{N}(I(i, j, k)-\tilde{I}(i, j, k))^{2}
$$

where $n_{g}$ is the number of gray-levels.

Tab. 2 displays the MSE scores for the different image sizes with 256 gray-level number. For all the tested resolu-

\begin{tabular}{|c|c|c|c|c|}
\hline Resolution & $16^{3}$ & $32^{3}$ & $64^{3}$ & $128^{3}$ \\
\hline MSE (\%) & $2.8 \pm 1.2$ & $2.7 \pm 1.2$ & $4.1 \pm 1.8$ & $14.2 \pm 7.4$ \\
\hline Exec. Time & $50 \mathrm{sec}$ & $20 \mathrm{~min}$ & 20 hours & 9 days \\
\hline
\end{tabular}

Table 2. Quantitative evaluation of the reconstruction performance on the phantom base in term of Mean Square Error.

tions except $128^{3}$, the error remains under $5 \%$ of wrong pixels. The worse result for the resolution $128^{3}$ is due to the high dimension of the research space. It shows the difficulty of the ab-initio reconstruction and our future work will exploit the lower resolution reconstructions to improve the result for higher resolution by including a multi-resolution process.

The robustness of the method against white noise has been evaluated. The proposed method has also been applied to real data composed of 225 averaged $160^{3}$ projections from the complex TAF7. Fig. 3 shows some examples of these classes. The signal to noise ratio is estimated at $10.02 \mathrm{~dB}$. The same protocol as for the phantom reconstruction has been used at

\footnotetext{
${ }^{1}$ Images and program are available at http://rhodes.unistra. $\mathrm{fr} / \mathrm{Tomo} / \mathrm{Data} / \mathrm{ISBI} 15 / \mathrm{DB} \_$resol_gr.zip
} 


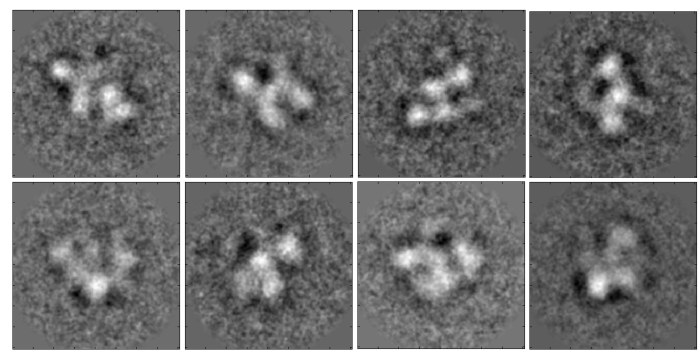

Fig. 3. Example of averaged projections of the complex TAF7.

the resolutions $40^{3}$ and $160^{3}$ but a positive result has been obtained only at the resolution $40^{3}$. A comparison is made with a reconstruction from the averaged projections with SIMPLE software [10]. Then the obtained ab-initio reconstructions are compared to a reference reconstruction obtained with a tilt acquisition. The MSE and the correlation are resp. 1.9784 and $85 \%$ for our method and resp $1.5 \times 10^{6}$ and $31 \%$ for SIMPLE software. It shows that our method obtains a better ab-initio reconstruction than the SIMPLE software on the complex TAF7. Fig. 4 shows isosurfaces for the obtained and the tilt volumes after registration.
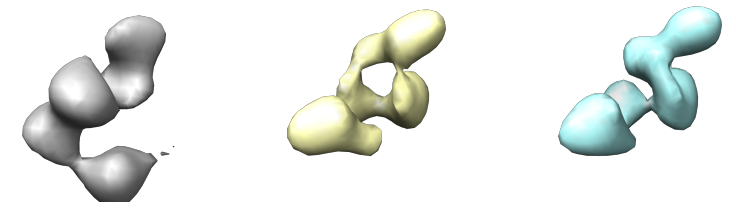

Fig. 4. Reconstructions of the complex TAF7 with SIMPLE software (left), with our method (center) and with the angular information from a tilt serie (right).

\section{CONCLUSION}

This paper presents a reconstruction method for the 3D case where the projection orientations are unknown. The method is based on the minimization of a cost depending on the image voxels and the orientations. The minimization uses a simulating annealing process. The proposed method has been tested on 20 synthetic images at 4 resolutions and on real data composed of projection averaged classes from the TAF7 complex at resolution $40^{3}$. The results are promising for the synthetic data and the reconstruction is successful on the real data and shows a good similarity with reference volume.

In the futur, we plan to introduce multiresolution in the method so as to speed up the process and to achieve reconstruction at higher resolutions.
Aknowledgement We thank Gabor Papai for providing the real data set, the reference volume and the SIMPLE reconstruction of the TAF7 complex.

\section{REFERENCES}

[1] R. A. Crowther, "Procedures for three-dimensional reconstruction of spherical viruses by Fourier synthesis from electron micrographs," Phils. Trans. R. Soc. Lond. $B$, vol. 261, no. 837, pp. 221-228, May 1971.

[2] J. Frank, Three-Dimensional Electron Microscopy of Macromolecular Assemblies, Oxford University Press, New York, 2006.

[3] P. A. Penczek, R. A. Grassucci, and J. Frank, "The ribosome at improved resolution: new techniques for merging and orientation refinement in $3 \mathrm{~d}$ cryo-electron microscopy of biological particles," Ultramicroscopy, vol. 53, no. 3, pp. 251-270, 1994.

[4] M. Van Heel, "Angular reconstitution: A posteriori assignment of projection directions for $3 \mathrm{~d}$ reconstruction," Ultramicroscopy, vol. 21, no. 2, pp. 111-123, 1987.

[5] R. R. Coifman, Y. Shkolnisky, F. J. Sigworth, and A. Singer, "Graph laplacian tomography from unknown random projections," IEEE T Image Process, vol. 17, no. 10, pp. 1891-1899, 2008.

[6] C. Yang, E. G. Ng, and P. A. Penczek, "Unified 3D structure and projection orientation refinement using quasi-Newton algorithm.," J Struct Biol, vol. 149, no. 1, pp. 53-64, Jan. 2005.

[7] T. Ogura and C. Sato, "A fully automatic 3d reconstruction method using simulated annealing enables accurate posterioric angular assignment of protein projections," $J$ Struct Biol, vol. 156, no. 3, pp. 371 - 386, 2006.

[8] M. van Heel, B. Gowen, R. Matadeen, E. V. Orlova, R. Finn, T. Pape, D. Cohen, et al., "Single-particle electron cryo-microscopy: towards atomic resolution," $Q$ Rev biophys, vol. 33, no. 04, pp. 307-369, 2000.

[9] B. Ben Cheikh, E. Baudrier, and G. Frey, "A tomographical reconstruction method from unknown direction projections for 2d gray-level images," in International Symposium on Biomedical Imaging. IEEE, Apr 2014.

[10] D. Elmlund and H. Elmlund, "Simple: Software for ab initio reconstruction of heterogeneous single-particles," J Struct Biol, vol. 180, no. 3, pp. 420 - 427, 2012. 